\title{
Black hole radiation with modified dispersion relation in tunneling paradigm: free-fall frame
}

\author{
Peng Wang ${ }^{\mathrm{a}}$, Haitang Yang ${ }^{\mathrm{b}}$, Shuxuan Ying ${ }^{\mathrm{c}}$ \\ Center for Theoretical Physics, College of Physical Science and Technology, Sichuan University, Chengdu 610064, People's Republic of China
}

Received: 21 May 2015 / Accepted: 18 December 2015 / Published online: 20 January 2016

(C) The Author(s) 2016. This article is published with open access at Springerlink.com

\begin{abstract}
Due to the exponential high gravitational red shift near the event horizon of a black hole, it might appear that the Hawking radiation would be highly sensitive to some unknown high energy physics. To study the effects of any unknown physics at the Planck scale on the Hawking radiation, the dispersive field theory models have been proposed, which are variations of Unruh's sonic black hole analogy. In this paper, we use the Hamilton-Jacobi method to investigate the dispersive field theory models. The preferred frame is the free-fall frame of the black hole. The dispersion relation adopted agrees with the relativistic one at low energy but is modified near the Planck mass $m_{p}$. The corrections to the Hawking temperature are calculated for massive and charged particles to $\mathcal{O}\left(m_{p}^{-2}\right)$ and neutral and massless particles with $\lambda=0$ to all orders. The Hawking temperature of radiation agrees with the standard one at the leading order. After the spectrum of radiation near the horizon is obtained, we use the brick wall model to compute the thermal entropy of a massless scalar field near the horizon of a 4D spherically symmetric black hole and a 2D one. Finally, the luminosity of a Schwarzschild black hole is calculated by using the geometric optics approximation.
\end{abstract}

\section{Contents}

1 Introduction . . . . . . . . . . . . 1

2 Deformed Hamilton-Jacobi method . . . . . . . . . 2

2.1 Deformed Hamilton-Jacobi equation . . . . . . 2

2.2 Massive and charged particle to $\mathcal{O}\left(m_{p}^{-2}\right) \ldots 3$

2.3 Massless and neutral particle to all orders . . . 4

2.4 Discussion................... 5

3 Entropy in brick wall model . . . . . . . . . . 5

\footnotetext{
a e-mail: pengw@scu.edu.cn

b e-mail: hyanga@scu.edu.cn

ce-mail: ysxuan@stu.scu.edu.cn
}

3.1 4D spherically symmetric black hole . . . . . 5

3.2 2D black hole in free-fall scenario . . . . . . 7

3.32 D black hole in static scenario . . . . . . . . 7

3.4 Discussion . . . . . . . . . . . . . . . 8

4 Black hole evaporation . . . . . . . . . . 10

5 Conclusion . . . . . . . . . . . . . 11

References . . . . . . . . . . . . . . . . . 12

\section{Introduction}

Soon after Hawking demonstrated that quantum effects could allow black holes to radiate a thermal flux of quantum particles [1], it was realized that there was the trans-Planckian problem with the calculation [2]. Hawking radiation appears to come from the modes with huge initial frequencies, well beyond the Planck mass $m_{p}$, which experience exponentially high gravitational red-shifting near the horizon. So the Hawking radiation relies on the validity of quantum field theory in curved spacetime to arbitrary high energies. On the other hand, quantum field theory is considered more like an effective field theory of an underlying theory whose nature remains unknown. This observation poses the question of whether any unknown physics at the Planck scale could strongly influence the Hawking radiation.

To study the trans-Planckian problem, a hydrodynamic analog of a black hole radiation was considered [3]. Following Unruh's work, there have been a lot of studies to understand the dispersive field theory models [3-13], which focused on studying the effect on the Hawking radiation due to modifications of the dispersion relations of matter fields at high energies. Similar to the original method for deriving the Hawking radiation, the energy fluxes for outgoing radiation were usually obtained by calculating the Bogoliubov transformations between the initial and final states of incoming and outgoing radiation. In most work, the Hawking effect could be recovered at leading order under some 
suitable assumptions, which have been briefly reviewed in $[14,15]$.

After the Hawking's original derivation, there have been some other methods proposed to understand the Hawking radiation. Recently, a semiclassical method of modeling Hawking radiation as a tunneling process has been developed and it has attracted a lot of attention. This method was first proposed by Kraus and Wilczek [16,17], which is known as the null geodesic method. They employed the dynamical geometry approach to calculate the imaginary part of the action for the tunneling process of s-wave emission across the horizon and related it to the Hawking temperature. Later, the tunneling behaviors of particles were investigated using the Hamilton-Jacobi method [18-20]. In the Hamilton-Jacobi method, one ignores the self-gravitation of the emitted particles and assumes that its action satisfies the relativistic Hamilton-Jacobi equation. The tunneling probability for the classically forbidden trajectory from inside to outside the horizon is obtained by using the HamiltonJacobi equation to calculate the imaginary part of the action for the tunneling process. Using the null geodesic method and Hamilton-Jacobi method has been very fruitful [21-32]. Furthermore, the effects of quantum gravity on the Hawking radiation have been discussed in the Hamilton-Jacobi method. In fact, the minimal length deformed Hamilton-Jacobi equation for fermions in curved spacetime have been introduced and the modified Hawking temperatures have been derived [33-38]. These have motivated us to use the Hamilton-Jacobi method to study the dispersive field theory models [39]. In this paper, we focus on the dispersive models with the freefall preferred fame, whereas those with the static preferred fame have been studied in [39].

The remainder of our paper is organized as follows. In Sect. 2, the deformed Hamilton-Jacobi equations are derived for the dispersive models with the free-fall preferred frame. We then solve the deformed Hamilton-Jacobi equations to obtain tunneling rates for massive and charged particles to $\mathcal{O}\left(m_{p}^{-2}\right)$ and massless and neutral particles to all orders. The thermal entropy of a massless scalar field near the horizon is computed in Sect. 3 using the brick wall model. In Sect. 4, we calculate the luminosity of a Schwarzschild black hole with the mass $M \gg m_{p}$. Section 5 is devoted to our conclusion. Throughout the paper we take geometrized units $c=G=1$, where the Planck constant $\hbar$ is the square of the Planck mass $m_{p}$.

\section{Deformed Hamilton-Jacobi method}

In this section, we first derive the deformed HamiltonJacobi equation incorporating the modified dispersion relation (MDR) assuming that the preferred reference frame is the free-fall frame. We then solve the deformed Hamilton-
Jacobi equation for the imaginary part of $I$, which gives the tunneling rate $\Gamma$ across the event horizon. We consider two cases, a massive and charged particle to $\mathcal{O}\left(m_{p}^{-2}\right)$ and a neutral and massless particle with $\lambda=0$ to all orders.

\subsection{Deformed Hamilton-Jacobi equation}

To study the deformed Hamilton-Jacobi method incorporating the MDR for the Hawking radiation, one first needs to choose the form of MDR in flat spacetime (the local free-fall frame) and generalizes it to curved spacetime. To be as general as possible, we will work with the MDR for a particle with mass $m$

$E^{2}=F^{2}(p)+m^{2}$

where we define

$F(p)=p \sum_{n=0} C_{n} \frac{p^{2 n}}{m_{p}^{2 n}}$,

$m_{p}$ is the Planck mass, $C_{0}=1$, and $E$ and $p$ are the energy and the norm of the momentum measured in some preferred reference frame, respectively. Note that the MDR (1) is rotational invariant in 4D spacetime. To generalize the MDR (1) to curved spacetime with the metric $g_{\mu \nu}$, we denote by $u^{\mu}$ the unit vector field tangent to the observers' world lines, which picks up a preferred frame. For a particle with the energy-momentum vector $p_{\mu}$, the energy $E$ and the norm of the momentum $p$ of the particle measured by these observers are

$$
\begin{aligned}
E & =p_{\mu} u^{\mu} \\
p^{2} & =E^{2}-p_{\mu} p^{\mu} .
\end{aligned}
$$

The curved spacetime generalization of the MDR (1) with a preferred frame described by $u^{\mu}$ is obtained by plugging Eqs. (3) and (4) into Eq. (1). To obtain the deformed HamiltonJacobi equation incorporating the MDR, one needs to relate the classical action $I$ to $p_{\mu}$. In fact, it can be shown that, if $I$ is a solution of the Hamilton-Jacobi equation, then the transformation equations give

$p_{\mu}=-\partial_{\mu} I$

where - appears since $p_{\mu}=(E,-\vec{p})$ in our metric signature. Replacing $p_{\mu}$ with $I$ via Eq. (5) and putting Eqs. (3) and (4) into Eq. (1) gives the deformed HamiltonJacobi equation. In the appendix of [39], the deformed Hamilton-Jacobi equation is also derived in the language of the effective field theory for a scalar field and a fermion one. There we considered a scalar/fermion with the mass $m$ and charge $q$ in a static black hole in the presence of an electromagnetic potential $A_{\mu}$. Neglecting self-interacting effective operators, we constructed the $U$ (1) gauge invariant 
effective field theory incorporating the MDR. The deformed Klein-Gordon/Dirac equation was derived. The deformed Hamilton-Jacobi equation for scalars/fermions was then obtained using the WKB approximation. It was found there that the deformed scalar/fermionic Hamilton-Jacobi equation with respect to the preferred frame $u^{\mu}$ in the black hole background spacetime can be written as

$T^{2}=F^{2}(X)+m^{2}$,

where

$T=-u^{\mu}\left(\partial_{\mu} I+q A_{\mu}\right), \quad X^{2}=T^{2}-\left(\partial_{\mu} I+q A_{\mu}\right)^{2}$,

$A_{\mu}$ is the black hole's electromagnetic potential and $q$ is the particle's charge.

As in [39], we here consider the black hole whose metric in the Schwarzschild-like coordinate is given by

$\mathrm{d} s^{2}=f(r) \mathrm{d} t^{2}-\frac{\mathrm{d} r^{2}}{f(r)}-C\left(r^{2}\right) h_{a b}(x) \mathrm{d} x^{a} \mathrm{~d} x^{b}$,

where $f(r)$ has a simple zero at $r=r_{h}$ with $f^{\prime}\left(r_{h}\right)$ being finite and nonzero. The vanishing of $f(r)$ at point $r=r_{h}$ indicates the presence of an event horizon. We also assumed that the black hole is asymptotically flat, which gives $f(r) \rightarrow 1$ as $r \rightarrow \infty$. However, a more suitable coordinate for describing a specific family of freely falling observers is the Painlevé-Gullstrand (PG) coordinate $[5,6,40]$. The PG coordinate anchored to the freely falling observers along the radial direction takes the form of

$\mathrm{d} s^{2}=\mathrm{d} t_{p}^{2}-\left[\mathrm{d} r-v(r) \mathrm{d} t_{p}\right]^{2}-C\left(r^{2}\right) h_{a b}(x) \mathrm{d} x^{a} \mathrm{~d} x^{b}$,

where $v(r)$ is the velocity of the free-fall observer with respect to the rest observer and $t_{p}$ measures proper time along them. The spacetime also has the event horizon at $r_{h}$ satisfying $v\left(r_{h}\right)=-1$. We assume $v<0, \mathrm{~d} v / \mathrm{d} r>0$, and $v \rightarrow v_{0} \leq 0$ as $r \rightarrow \infty$. Note that $v<0$ means the infalling observers. For simplicity we specialize to the particular family of observers with $v_{0}=0$ who start at infinity with a zero initial velocity. Since the vector field $u^{\mu}$ of the freely falling observers is tangent to the infalling world lines, one has for the infalling observers along the radial direction with $v_{0}=0$

$u^{\mu}=(1, v(r), \overrightarrow{0})$

in the PG coordinate and

$u^{\mu}=\left(\frac{1}{f(r)}, \sqrt{1-f(r)}, \overrightarrow{0}\right)$

in the Schwarzschild-like coordinate. The fact that $t_{p}$ is the proper time along the infalling world lines means that $u_{\mu}$ is equal to the gradient of $t_{p}$,

$u_{\mu}=\partial_{\mu} t_{p}$.
Using Eqs. (11) and (12) gives

$t_{p}=t+\int \frac{\sqrt{1-f(r)}}{f(r)} \mathrm{d} r$.

Substituting Eq. (13) into the PG metric (9) and comparing it to the Schwarzschild-like coordinate (8), one finds

$v(r)=-\sqrt{1-f(r)}$.

In the PG coordinate, one can use Eq. (10) to show that Eq. (7) becomes

$$
\begin{aligned}
T & =-\left(\partial_{t} I+q A_{t}\right)-v(r)\left(\partial_{r} I+q A_{r}\right), \\
X^{2} & =\left(\partial_{r} I+q A_{r}\right)^{2}+\frac{h^{a b}(x)\left(\partial_{a} I+q A_{a}\right)\left(\partial_{b} I+q A_{b}\right)}{C\left(r^{2}\right)} .
\end{aligned}
$$

Note that $\partial_{t}$ and $\partial_{t_{p}}$ are Killing vectors in the Schwarzschild-like coordinate and the PG coordinate, respectively. For the energy-momentum vector $p_{\mu}$, Eq. (13) shows that its Killing energies associated with $\partial_{t}$ and $\partial_{t_{p}}$ are the same. Let $\omega$ denote the Killing energies in the PG and Schwarzschildlike coordinates. Explicitly, one has $\omega=\partial_{t}^{\mu} p_{\mu}=\partial_{t_{p}}^{\mu} p_{\mu}$, which is a constant.

2.2 Massive and charged particle to $\mathcal{O}\left(m_{p}^{-2}\right)$

Since $\omega=-\partial_{t_{p}} I$ is the conserved energy of the particle, we can separate $t$ from other variables. Thus, we employ the following ansatz for the action $I$ :

$I=-\omega t+W(r)+\Theta(x)$.

The vector potential $A_{\mu}$ is assumed to be given by

$A_{\mu}=A_{t}(r) \delta_{\mu t}$

which is true for charged static black holes in most cases. Putting the ansatz (16) into Eq. (15), we have

$$
\begin{aligned}
T & =\tilde{\omega}(r)-v(r) p_{r} \\
X^{2} & =p_{r}^{2}+\frac{h^{a b}(x) \partial_{a} \Theta(x) \partial_{b} \Theta(x)}{C\left(r^{2}\right)},
\end{aligned}
$$

where $\tilde{\omega}(r)=\omega-q A_{t}(r)$ and $p_{r}=\partial_{r} W$. The method of separation of variables gives the differential equation for $\Theta(x)$

$h^{a b}(x) \partial_{a} \Theta(x) \partial_{b} \Theta(x)=\lambda$,

where is $\lambda$ is a constant and determined by $h^{a b}(x)$. Thus, one has

$X^{2}=p_{r}^{2}+\frac{\lambda}{C\left(r^{2}\right)}$, 
and Eq. (6) becomes an ordinary differential equation for $W(r)$.

We consider a particle with mass $m$ and charge $q$. Solving Eq. (6) for $p_{r}$ gives

$$
\begin{aligned}
p_{r}^{ \pm}= & \frac{ \pm \sqrt{\Lambda}+\sqrt{1-f}}{f} \tilde{\omega}(r) \mp \frac{C_{1}}{m_{p}^{2}} \frac{[1 \pm \sqrt{(1-f) \Lambda}]^{4} \tilde{\omega}^{3}(r)}{f^{4} \sqrt{\Lambda}} \\
& +\mathcal{O}\left(m_{p}^{-4}\right)
\end{aligned}
$$

where $+/-$ denotes the outgoing/ingoing solutions and $\Lambda=$ $1-\frac{m^{2}+\frac{\lambda}{C\left(r^{2}\right)}}{\tilde{\omega}^{2}(r)}$. Here, $p_{r}^{+}$has a pole at $r=r_{h}$. To obtain the residue of $p_{r}^{+}$at $r=r_{h}$, one expands $f(r)$ and $C\left(r^{2}\right)$ at $r=r_{h}$

$$
\begin{aligned}
& f(r) \sim 2 \kappa\left(r-r_{h}\right) \\
& \quad \times\left[1+\eta \kappa\left(r-r_{h}\right)+\theta \kappa^{2}\left(r-r_{h}\right)^{2}+\rho \kappa^{3}\left(r-r_{h}\right)^{3}\right], \\
& C\left(r^{2}\right) \sim C\left(r_{h}^{2}\right) \\
& \quad \times\left[1+c_{1} \kappa\left(r-r_{h}\right)+c_{2} \kappa^{2}\left(r-r_{h}\right)^{2}+c_{3} \kappa^{3}\left(r-r_{h}\right)^{3}\right], \\
& \tilde{\omega}(r) \sim \tilde{\omega}\left(r_{h}\right) \\
& \quad \times\left[1+\omega_{1} \kappa\left(r-r_{h}\right)+\omega_{2} \kappa^{2}\left(r-r_{h}\right)^{2}+\omega_{3} \kappa^{3}\left(r-r_{h}\right)^{3}\right]
\end{aligned}
$$

where $\kappa=f^{\prime}\left(r_{h}\right) / 2$. Using the residue theory for the semi circles, we get

$\operatorname{Im} W_{+}(r)=\frac{\tilde{\omega}\left(r_{h}\right) \pi}{\kappa}(1+\Delta)$,

where we define

$$
\begin{aligned}
\Delta= & \frac{C_{1}}{2 m_{p}^{2}}\left[\frac{\delta_{\lambda} \lambda}{C\left(r_{h}^{2}\right)}+\delta_{m} m^{2}+\delta_{\omega} \tilde{\omega}^{2}\left(r_{h}\right)\right]+\mathcal{O}\left(m_{p}^{-4}\right), \\
\delta_{\lambda}= & 3+2 c_{1}^{2}-2 c_{2}+12 \eta+12 \eta^{2}-6 \theta+c_{1}\left(6+6 \eta-2 \omega_{1}\right) \\
& -6 \omega_{1}-6 \eta \omega_{1}+2 \omega_{2}, \\
\delta_{m}= & -1+12 \eta^{2}-6 \theta+\eta\left(4-6 \omega_{1}\right)-2 \omega_{1}+2 \omega_{2}, \\
\delta_{\omega}= & 40 \eta^{3}-12 \theta+8 \rho+\eta^{2}\left(24-60 \omega_{1}\right)-3 \omega_{1}+24 \theta \omega_{1}+12 \omega_{1}^{2} \\
& -2 \omega_{1}^{2}+12 \omega_{2}-12 \omega_{1} \omega_{2}+\eta \\
& \times\left(2-40 \theta-36 \omega_{1}+24 \omega_{1}^{2}+24 \omega_{2}\right)-6 \omega_{3} .
\end{aligned}
$$

On the other hand, one can use Eq. (22) to expand $p_{r}^{-}$ at $r=r_{h}$. It turns out that the residue of $p_{r}^{-}$at $r=r_{h}$ is zero. Hence, we have $\operatorname{Im} W_{-}(r)=0$. As shown in [39], the probability of a particle tunneling from inside to outside the horizon is

$P_{\text {emit }} \propto \exp \left[-\frac{2}{\hbar}\left(\operatorname{Im} W_{+}-\operatorname{Im} W_{-}\right)\right]$.

There is a Boltzmann factor in $P_{\text {emit }}$ with an effective temperature, which is

$T_{\text {eff }}=\frac{T_{0}}{1+\Delta}$.
It is interesting to note that we have calculated $\Delta$ in the static preferred frame in [39]. For emitted particles with mass $m$ and charge $q$, we found

$$
\Delta=-\frac{C_{1}}{2 m_{p}^{2}}\left[2\left(3 \omega_{1}-\eta\right) \tilde{\omega}^{2}\left(r_{h}\right)+\frac{\lambda}{C\left(r_{h}^{2}\right)}+m^{2}\right]+\mathcal{O}\left(m_{p}^{-4}\right) .
$$

\subsection{Massless and neutral particle to all orders}

We now work with a particle with $m=0$ and $q=0$. To get an all-order result, we let $\lambda=0$. Note that one has $\lambda=0$ for the solution in a 2D black hole or the s-wave solution in a 4D spherically symmetric black hole. In this case, we can use the following ansatz for the action $I$ :

$I=-\omega t+W(r)$.

Hence, the deformed Hamilton-Jacobi equation becomes

$\left(\omega-v p_{r}\right)^{2}=p_{r}^{2}\left(\sum_{n=0} C_{n} \frac{p_{r}^{2 n}}{m_{p}^{2 n}}\right)^{2}$,

where $p_{r}=\partial_{r} W$. We will prove by induction that the solutions to Eq. (29) take the form of

$p_{r}^{ \pm}=\frac{ \pm \omega}{1 \pm v}\left[1+\sum_{i=1}^{\infty} \frac{\omega^{2 i}}{m_{p}^{2 i}} \sum_{j=0}^{i-1} \frac{C_{i, j}}{(1 \pm v)^{3 i-j}}\right]$

where $C_{i, j}$ is determined by $C_{n}$. In fact, it is easy to see that

$$
\begin{aligned}
p_{r}^{ \pm} & =\frac{ \pm \omega}{1 \pm v} \\
& \times\left[1-\frac{C_{1} \omega^{2}}{m_{p}^{2}(1 \pm v)^{3}}+\frac{\omega^{4}\left[3 C_{1}^{2}-C_{2}(1 \pm v)\right]}{m_{p}^{4}(1 \pm v)^{6}}+\mathcal{O}\left(\frac{\omega^{6}}{m_{p}^{6}}\right)\right] .
\end{aligned}
$$

Suppose for some integer $N>1$ that

$p_{r, N}^{ \pm}=\frac{ \pm \omega}{1 \pm v}\left[1+\sum_{i=1}^{N} \frac{\omega^{2 i}}{m_{p}^{2 i}} \sum_{j=0}^{i-1} \frac{C_{i, j}}{(1 \pm v)^{3 i-j}}\right]$

satisfy Eq. (29) at $\mathcal{O}\left(\frac{\omega^{2 N}}{m_{p}^{2 N}}\right)$. Plugging $p_{r}=p_{r, N}^{ \pm}+$ $\frac{\alpha^{ \pm} \omega^{2 N+2}}{m_{p}^{2 N+2}} \frac{\omega}{1 \pm v}$ into Eq. (29), one finds that the terms of $\mathcal{O}\left(\frac{\omega^{2 N+2}}{m_{p}^{2 N+2}}\right)$ gives

$\alpha^{ \pm}=\frac{P_{N-1}(1 \pm v)}{(1 \pm v)^{3(N+1)}}$

where $P_{N-1}(x)$ is some polynomial of $x$ with degree $N-1$. This completes the proof that Eq. (30) is a solution to Eq. (29) to all orders. We define the residue of $\frac{1}{(1+v)^{n}}$ at $r=r_{h}$ as 
$\operatorname{Res}\left(\frac{1}{(1+v)^{n}}, r_{h}\right)=\frac{R_{n}}{\kappa}$,

where $R_{1}=1$. Thus, one obtains

$\operatorname{Im} W^{+}=\frac{\omega \pi}{\kappa}(1+\Delta), \quad \operatorname{Im} W^{-}=0$,

where we define

$\Delta=\sum_{i=1}^{\infty} \frac{\delta_{i} \omega^{2 i}}{m_{p}^{2 i}} \quad$ with $\quad \delta_{i}=\left(\sum_{j=0}^{i-1} C_{i, j} R_{3 i+1-j}\right)$.

Using Eq. (25) gives the effective temperature

$T_{\text {eff }}=\frac{T_{0}}{1+\Delta}$.

\subsection{Discussion}

When we use the residue theory for the semi circles to give Eqs. (23) and (35), an assumption proposed in [39] is needed. The assumption requires that the singularity structure of $\partial_{r} I$ except the order of the pole at $r=r_{h}$ do not change after the MDR is introduced. It follows that $\omega \lesssim m_{p}$. A complete theory of quantum gravity might been needed to justify this assumption.

In most works of the dispersive models, much attention have been paid to the modifications of the asymptotic spectrum. Since the tunneling across the horizon takes place near the horizon, the near horizon spectrum of radiations is computed in the paper. The effects of scattering off the background need to be included if the asymptotic spectrum at infinity is considered. In most works, 2D spacetime has been considered. The higher order terms in the MDR violate conformal invariance of 2D spacetime, hence there is some scattering. Our calculations show that the spectrum of radiation near the horizon is close to a perfect thermal spectrum in the dispersive models. The thermal asymptotic spectrum has been recovered at leading order in previous studies. Thus, the energy fluxes of radiations are not significantly affected by the effects of scattering off the background. However, as noted in [39], the scattering effects might dramatically change the spectrum of radiations in the dispersive models with the static preferred frame.

\section{Entropy in brick wall model}

Bekenstein and Hawking showed that the entropy of a black hole is proportional to the area of the horizon [41-43]. Although all the evidence suggests that the BekensteinHawking entropy is the thermodynamic entropy, the statistical origin of the black hole entropy is not yet fully understood. It appears that an unavoidable candidate for the statistical origin is the entropy of the thermal atmosphere of the black hole.
However, the entropy diverges when we attempt to calculate the entropy of the thermal atmosphere. There are two kinds of divergences. The first one is due to infinite volume of the system, which has to do with the contribution from the vacuum surrounding the system at large distances and is of little relevance here. The second one arises from the infinite volume of the deep throat region near the horizon. To regulate the divergences, 't Hooft [44] proposed the brick wall model for a scalar field $\phi$, where two brick wall cutoffs are introduced at some small distance $r_{\varepsilon}$ from the horizon and at a large distance $L \gg r_{h}$,

$\phi=0 \quad$ at $r=r_{h}+r_{\varepsilon} \quad$ and $r=L$.

In the following, we will use the brick wall model to calculate the entropy of a scalar field for a 4D spherically symmetric black hole and a 2D one. For a 4D spherically symmetric black hole, the entropy will be calculated to $O\left(m_{p}^{-2}\right)$. For the 2D black hole, we will obtain all order results in the cases with the static and free-fall preferred frames for comparison. For simplicity, we assume that the scalar field is massless and neutral.

\subsection{D spherically symmetric black hole}

For a 4D spherically symmetric black hole with the Schwarzschild-like coordinate

$\mathrm{d} s^{2}=f(r) \mathrm{d} t^{2}-\frac{\mathrm{d} r^{2}}{f(r)}-C\left(r^{2}\right)\left(\mathrm{d} \theta^{2}+\sin ^{2} \theta \mathrm{d} \phi^{2}\right)$,

we have shown that $\lambda=\left(l+\frac{1}{2}\right)^{2} \hbar^{2}$ with the angular momentum $l=0,1, \ldots$ and the corresponding degeneracy is $2 l+1$ [39]. Thus, the atmosphere entropy of a massless scalar field can be expressed in the form of

$S=\int(2 l+1) \mathrm{d} l \int \mathrm{d} \omega \frac{\mathrm{d} n(\omega, l)}{\mathrm{d} \omega} s_{\omega, l}$,

where $\omega$ is the Killing energy associated with $t, l$ is the angular momentum, $n(\omega, l)$ is the number of one-particle states not exceeding $\omega$ with fixed value of angular momentum $l$, and $s_{\omega, l}$ is the thermal entropy per mode. Taking the MDR corrections to both $n(\omega, l)$ and the Hawking temperature into consideration, we used the brick wall model to calculate this entropy to all orders in [39], where the static preferred frame is used to import a MDR to the black hole background. By contrast, here the MDR corrected atmosphere entropy of the black hole is computed to $\mathcal{O}\left(m_{p}^{-2}\right)$ in the free-fall scenario.

For particles emitted in a wave mode labeled by energy $\omega$ and $l$, we find that 
(Probability for a black hole to emit a particle in this mode)

$$
\begin{aligned}
& =\exp \left(-\frac{\omega}{T_{\text {eff }}}\right) \times(\text { Probability for a black hole to absorb } \\
& \text { a particle in the same mode), }
\end{aligned}
$$

where $T_{\text {eff }}$ is given by Eq. (26). The above relation for the usual dispersion relation was obtained by Hartle and Hawking [48] using a semiclassical analysis. Neglecting backreaction, detailed balance condition requires that the ratio of the probability of having $N$ particles in a particular mode to the probability of having $N-1$ particles in the same mode is $\exp \left(-\frac{\omega}{T_{\text {eff }}}\right)$. One then follows the argument in [39] to get the average number $n_{\omega, l}$ in the mode

$n_{\omega, l}=n\left(\frac{\omega}{T_{\mathrm{eff}}}\right)$,

where we define

$n(x)=\frac{1}{\exp x-(-1)^{\epsilon}}$.

Note that $\epsilon=0$ for bosons and $\epsilon=1$ for fermions. The von Neumann entropy for the mode is

$s_{\omega, l}=\left[n_{\omega, l}+(-1)^{\epsilon}\right] \ln \left[1+(-1)^{\epsilon} n_{\omega, l}\right]-n_{\omega, l} \ln n_{\omega, l}$.

Moreover, the entropy per mode $s_{\omega, l}$ can be put in the form of

$s_{\omega, l}=s\left(\frac{\omega}{T_{\mathrm{eff}}}\right)$,

where the $s(x)$ is given by

$$
\begin{aligned}
s(x)= & \frac{(-1)^{\epsilon} \exp x}{\exp x-(-1)^{\epsilon}} \ln \left[\frac{\exp x}{\exp x-(-1)^{\epsilon}}\right] \\
& +\frac{\ln \left[\exp x-(-1)^{\epsilon}\right]}{\exp x-(-1)^{\epsilon}} .
\end{aligned}
$$

Defining $u=\frac{\omega}{T_{0}}$ and expanding $s_{\omega, l}$ to $\mathcal{O}\left(m_{p}^{-2}\right)$ give

$s_{\omega, l} \approx s(u)+s^{\prime}(u) u \Delta$,

where one has

$\Delta=\frac{C_{1} \delta_{\lambda} z}{2}+\frac{C_{1} \delta_{\omega} T_{0}^{2}}{2 m_{p}^{2}} u^{2}$.

In the brick wall model, 't Hooft found that the number of one-particle states not exceeding the $\omega$ fixed value $l$ is

$n(\omega, l)=\frac{1}{2 \pi \hbar} \oint p_{r} \mathrm{~d} r$

where the integral $\oint p_{r} \mathrm{~d} r$ was calculated in the Schwarzschild-like coordinate. Nevertheless, we calculate $p_{r}^{ \pm}$in the PG coordinate in Sect. 2. In $[29,47]$, the integral $\oint p_{r} \mathrm{~d} r$ has been found to be invariant under canonical transformations. Hence, the number of states $n(\omega, l)$ given in Eq. (48) is the same in the Schwarzschild-like and PG coordinates and one does not need to re-calculate it in different coordinates. Define the radial wave number $k(r, l, \omega)$ by

$k^{ \pm}(r, l, \omega)=p_{r}^{ \pm}$,

as long as $p_{r}^{ \pm 2} \geq 0$, and $k^{ \pm}(r, l, \omega)=0$ otherwise. With these two Dirichlet boundaries, one finds that the number of one-particle states not exceeding the $\omega$ fixed value $l$ is

$n(\omega, l)=\frac{1}{2 \pi \hbar}\left[\int_{r_{h}+r_{\varepsilon}}^{L} k^{+}(r, l, \omega) \mathrm{d} r+\int_{L}^{r_{h}+r_{\varepsilon}} k^{-}(r, l, \omega) \mathrm{d} r\right]$.

The $p_{r}^{ \pm}$in Eq. (49) are given by Eq. (21) in Sect. 2. For a massless and neutral scalar field, one thus has

$$
\begin{aligned}
p_{r}^{ \pm}= & \frac{ \pm \sqrt{\Lambda}+\sqrt{1-f}}{f} \omega \mp \frac{C_{1}}{m_{p}^{2}} \frac{[1 \pm \sqrt{\Lambda(1-f)}]^{4} \omega^{3}}{f^{4} \sqrt{\Lambda}} \\
& +\mathcal{O}\left(m_{p}^{-4}\right)
\end{aligned}
$$

where $\Lambda=1-f \frac{\left(l+\frac{1}{2}\right)^{2} \hbar^{2}}{C\left(r^{2}\right) \omega^{2}}$.

Integrating by parts, one finds the entropy becomes

$S=-\frac{1}{\pi \hbar} \int(2 l+1) \mathrm{d} l \int \mathrm{d} u \frac{\partial s_{\omega, l}}{\partial u} \int_{r_{h}+r_{\varepsilon}}^{L} k(r, l, \omega) \mathrm{d} r$,

where we define $k(r, l, \omega)=\frac{k^{+}(r, l, \omega)-k^{-}(r, l, \omega)}{2}$. Plugging Eqs. (51) and (46) into Eq. (52) and performing the $l$ integral which runs over the region where $\Lambda>0$, we find that the entropy to $\mathcal{O}\left(m_{p}^{-2}\right)$ becomes

$$
\begin{aligned}
S \approx & \frac{C\left(r_{h}^{2}\right) T_{0}^{3}}{\pi \hbar^{3}} \int u^{2} \mathrm{~d} u \int_{r_{h}+r_{\varepsilon}}^{L} \frac{C\left(r^{2}\right)}{C\left(r_{h}^{2}\right)} \frac{\mathrm{d} r}{f^{2}}\{2 s(u) \\
& +\frac{C_{1} \delta_{\omega} T_{0}^{2}}{m_{p}^{2}} u^{3} s^{\prime}(u)+\frac{2 C_{1} \delta_{\lambda} C\left(r^{2}\right) T_{0}^{2}}{3 f C\left(r_{h}^{2}\right) m_{p}^{2}} u^{3} s^{\prime}(u) \\
& \left.-\frac{5 C_{1} T_{0}^{2} u^{2} s(u)}{2 m_{p}^{2} f^{3}}\left(12-\frac{48 f}{5}+\frac{f^{2}}{2}-\frac{7 f^{4}}{32}\right)\right\},
\end{aligned}
$$

where second and third terms in the bracket come from the MDR corrections to the Hawking temperature and the fourth term from the MDR corrections to $n(\omega, l)$. Focusing on the divergent parts near horizon, we obtain for the nonnegative integers $a$ and $n$,

$$
\begin{aligned}
& \int_{r_{h}+r_{\varepsilon}} \frac{C^{a}\left(r^{2}\right)}{C^{a}\left(r_{h}^{2}\right)} \frac{\mathrm{d} r}{f(r)^{n+1}} \sim \frac{1}{2^{n+1} \kappa} \sum_{k=0}^{n-1} \frac{\tilde{f}_{k}^{n, a}}{n-k}\left(\kappa r_{\varepsilon}\right)^{k-n} \\
& -\frac{\tilde{f}_{n}^{n, a}}{2^{n+1} \kappa} \ln \kappa r_{\varepsilon},
\end{aligned}
$$


where we expand $f(r)^{-n}$ and $\frac{C^{a}\left(r^{2}\right)}{C^{a}\left(r_{h}^{2}\right)}$ at $r=r_{h}$

$f(r)^{-n}=2^{-n} \kappa^{-n}\left(r-r_{h}\right)^{-n} \sum_{j=0}^{\infty} f_{j}^{n} \kappa^{j}\left(r-r_{h}\right)^{j}$,

$\frac{C^{a}\left(r^{2}\right)}{C^{a}\left(r_{h}^{2}\right)}=\sum_{i=0} c_{i}^{a} \kappa^{i}\left(r-r_{h}\right)^{i}$,

and we define $\tilde{f}_{k}^{n, a}=\sum_{j=0}^{k} f_{j}^{n+1} c_{k-j}^{a}$. In Eq. (54), we neglect finite terms as $\kappa r_{\varepsilon} \rightarrow 0$ and terms involving $L$. Note that we define $\kappa=\frac{f^{\prime}\left(r_{h}\right)}{2}$, which is the surface gravity for the black hole and hence $T_{0}=\frac{\hbar \kappa}{2 \pi}$. Thus, the divergent part of entropy near the horizon to $\mathcal{O}\left(m_{p}^{-2}\right)$ is

$$
\begin{aligned}
S \sim & \frac{C\left(r_{h}^{2}\right) \kappa^{2}}{16 \pi^{4}} \int s(u) u^{2} \mathrm{~d} u\left(\frac{1}{\kappa r_{\varepsilon}}-\left(c_{1}-2 \eta\right) \ln \kappa r_{\varepsilon}\right) \\
& -\frac{C\left(r_{h}^{2}\right) \kappa^{2}}{16 \pi^{4}} \frac{m_{p}^{2} \kappa^{2}}{4 \pi^{2}} \int s(u) u^{4} \mathrm{~d} u\left\{\frac{5 C_{1} \delta_{\omega}}{2}\left(\frac{\tilde{f}_{0}^{1,1}}{\kappa r_{\varepsilon}}-\tilde{f}_{1}^{1,1} \ln \kappa r_{\varepsilon}\right)\right. \\
& +\frac{5 C_{1} \delta_{\lambda}}{6}\left[\sum_{k=0}^{1} \frac{\tilde{f}_{k}^{2,2}}{2-k}\left(\kappa r_{\varepsilon}\right)^{k-2}-\tilde{f}_{2}^{2,2} \ln \kappa r_{\varepsilon}\right] \\
& +5 C_{1}\left[\frac{12}{32} \sum_{k=0}^{3} \frac{\tilde{f}_{k}^{4,1}}{4-k}\left(\kappa r_{\varepsilon}\right)^{k-4}-\frac{3}{5} \sum_{k=0}^{2} \frac{\tilde{f}_{k}^{3,1}}{3-k}\left(\kappa r_{\varepsilon}\right)^{k-3}+\frac{\tilde{f}_{0}^{1,1}}{8 \kappa r_{\varepsilon}}\right. \\
& \left.\left.+\left(\frac{7 \tilde{f}_{0}^{0,1}}{64}-\frac{\tilde{f}_{1}^{1,1}}{8}+\frac{3 \tilde{f}_{3}^{3,1}}{5}-\frac{12 \tilde{f}_{4}^{4,1}}{32}\right) \ln \kappa r_{\varepsilon}\right]\right\} .
\end{aligned}
$$

\subsection{D black hole in free-fall scenario}

Consider a 2D black hole with the metric of

$\mathrm{d} s^{2}=f(r) \mathrm{d} t^{2}-\frac{\mathrm{d} r^{2}}{f(r)}$,

in the Schwarzschild-like coordinate. The atmosphere entropy of a massless scalar field is

$S=\int \mathrm{d} \omega \frac{\mathrm{d} n(\omega, l)}{\mathrm{d} \omega} s_{\omega}$,

where $s_{\omega}$ is the entropy per mode. Define the radial wave number $k(r, \omega)$ by

$k^{ \pm}(r, \omega)=p_{r}^{ \pm}$,

as long as $p_{r}^{ \pm 2} \geq 0$, and $k^{ \pm}(r, \omega)=0$ otherwise. The $p_{r}^{ \pm}$ in Eq. (59) are given in Eq. (30) in Sect. 2. The number of one-particle states not exceeding $\omega$ is

$n(\omega)=\frac{1}{2 \pi \hbar} \int_{r_{h}+r_{\varepsilon}}^{L}\left(k_{r}^{+}-k_{r}^{-}\right) \mathrm{d} r$.

Defining the coefficients $\sigma_{s}^{q}$ by

$\Delta^{q}=\left(\frac{m_{p} \kappa}{2 \pi}\right)^{2 q} u^{2 q} \sum_{s=0}^{\infty} \sigma_{s}^{q}\left(\frac{m_{p} \kappa}{2 \pi}\right)^{2 s} u^{2 s}$, one has for $s_{\omega}$

$$
\begin{aligned}
s_{\omega} & =s\left(\frac{\omega}{T_{\mathrm{eff}}}\right) \\
& =\sum_{r=0}^{\infty} \sum_{q=0}^{r} \frac{s^{(q)}(u) u^{q+2 r} \sigma_{r-q}^{q}}{q !}\left(\frac{m_{p} \kappa}{2 \pi}\right)^{2 r},
\end{aligned}
$$

where $u=\frac{\omega}{T_{0}}$ and we use Eq. (37) for $T_{\text {eff }}$. Expanding $(1+v)^{i}$ at $r=r_{h}$

$$
(1+v)^{i}=\kappa^{i}\left(r-r_{h}\right)^{i} \sum_{j=0}^{\infty} v_{j}^{i} \kappa^{j}\left(r-r_{h}\right)^{j},
$$

we find the divergent part of entropy near the horizon becomes

$$
\begin{aligned}
S \sim & -\frac{1}{4 \pi^{2}} \ln \kappa r_{\varepsilon} \sum_{r=0}^{\infty} \sum_{i=0}^{\infty} \sum_{q=0}^{r} \frac{(2 i+1) \eta_{i} \sigma_{r-q}^{q}}{q !}\left(\frac{m_{p} \kappa}{2 \pi}\right)^{2 i+2 r} \\
& \times \int \mathrm{d} u s^{(q)}(u) u^{q+2 r+2 i} \\
& +\frac{1}{4 \pi^{2}} \sum_{l=1}^{\infty} \frac{1}{l\left(\kappa r_{\varepsilon}\right)^{l}}\left(\frac{m_{p} \kappa}{2 \pi}\right)^{2\lceil l / 3\rceil} \sum_{r=0}^{\infty} \sum_{i=0}^{\infty} \sum_{q=0}^{r} \frac{\xi_{i, l} \sigma_{r-q}^{q}}{q !} \\
& \times\left(\frac{m_{p} \kappa}{2 \pi}\right)^{2 i+2 r} \int \mathrm{d} u s^{(q)}(u) u^{q+2 r+2 i+2\lceil l / 3\rceil},
\end{aligned}
$$

where $\lceil x\rceil$ is the smallest integer that is not less than $x$ and we define

$$
\begin{aligned}
\eta_{i>0}= & \sum_{j=0}^{i-1} C_{i, j} v_{3 i-j}^{-3 i+j-1} \text { and } \eta_{0}=1, \\
\xi_{i, l}= & \sum_{j=0}^{3 i+3[l / 3]^{+}-l}\left(2 i+2[l / 3]^{+}+1\right) \\
& \times C_{i+[l / 3]^{+}, j} v_{3 i-j-l+3[l / 3]^{+}}^{-l}
\end{aligned}
$$

\subsection{D black hole in static scenario}

Following the conventions adopted in [39], the MDR for a massless scalar particle considered here takes the form of

$p^{2}=\tilde{F}^{2}(E)$,

where we define

$\tilde{F}(E)=E \sum_{n=0}^{\infty} \frac{\tilde{C}_{n} E^{2 n}}{m_{p}^{2 n}}$,

with $\tilde{C}_{0}=1$. For a particle with the energy-momentum vector $p_{\mu}$, the energy $\omega$ and the norm of the momentum $p$ of the particle measured by the static observers hovering above the 2D black hole with the metric (57) are 


$$
E=\frac{p_{t}}{\sqrt{f(r)}}
$$

$p^{2}=f(r) p_{r}^{2}$.

Relating $p_{\mu}$ to the action $I$ by $p_{\mu}=-\partial_{\mu} I$ gives the deformed Hamilton-Jacobi equation

$X^{2}=\tilde{F}^{2}(T)$,

where

$T=\frac{\omega}{\sqrt{f(r)}}, \quad X=\sqrt{f(r)} p_{r}$,

and $\omega=\partial_{t}^{\mu} p_{\mu}$ is the Killing energy with respect to $t$. Solving Eq. (69) for $p_{r}$, one could define the radial wave number $k(r, \omega)$ by

$k(r, \omega)=\left|p_{r}\right|$,

as long as $p_{r}^{2} \geq 0$, and $k(r, \omega)=0$ otherwise. The number of one-particle states not exceeding $\omega$ is

$n(\omega)=\frac{1}{\pi \hbar} \int_{r_{h}+r_{\varepsilon}}^{L} k_{r} \mathrm{~d} r$.

The entropy per mode $s_{\omega}$ is

$s_{\omega}=s\left(\frac{\omega}{T_{\text {eff }}}\right)$,

where $s(x)$ is given in Eq. (45) and $T_{\text {eff }}$ is the effective Hawking temperature. We calculated $T_{\text {eff }}$ in [39] and it was given by

$T_{\text {eff }}=\frac{T_{0}}{1+\Delta}$,

where one has

$\Delta=\sum_{k=1}^{\infty} \eta_{0}^{2 k} \zeta_{k}^{0} \frac{\omega^{2 k}}{m_{p}^{2 k}}$

and $\eta_{0}^{2 k}$ and $\zeta_{k}^{0}$ are defined in [39]. Defining the coefficients $\tilde{\sigma}_{s}^{q}$ by

$\tilde{\Delta}^{q}=\left(\frac{m_{p} \kappa}{2 \pi}\right)^{2 q} u^{2 q} \sum_{s=0}^{\infty} \tilde{\sigma}_{s}^{q}\left(\frac{m_{p} \kappa}{2 \pi}\right)^{2 s} u^{2 s}$,

we find the divergent part of entropy near the horizon becomes

$$
\begin{aligned}
S \sim & \frac{1}{4 \pi^{2}} \sum_{n=0}^{\infty} \sum_{r=0}^{\infty} \sum_{q=0}^{r}\left(\frac{m_{p} \kappa}{2 \pi}\right)^{2 n+2 r} \frac{2 n+1}{2^{n}} \frac{\tilde{\sigma}_{r-q}^{q}}{q !} \tilde{C}_{n} \\
& \times\left(\sum_{l=1}^{n} \frac{\tilde{f}_{n-l}^{n, 0}}{l\left(\kappa r_{\varepsilon}\right)^{l}}-\tilde{f}_{n}^{n, 0} \ln r_{\varepsilon}\right) \int u^{2 n+2 r+q} S^{(q)}(u) \mathrm{d} u,
\end{aligned}
$$

where $\tilde{f}_{k}^{n, a}$ with $n \geq k \geq 0$ are defined in Eq. (54).

\subsection{Discussion}

In [39] and this paper, we have calculated the divergent part of the near horizon atmosphere entropy of a massless scalar field for a 4D spherically symmetric black hole in the static and free-fall scenarios, respectively. It appears that the divergent part in both scenarios can be presented in the form of a Laurent series with respect to $r_{\varepsilon}$

$S \sim \frac{s_{1}^{0}}{\kappa r_{\varepsilon}}+s_{0}^{0} \ln \kappa r_{\varepsilon}+\sum_{i=1}^{\infty} \frac{T_{0}^{2 i}}{m_{p}^{2 i}}\left(\sum_{j=1}^{\delta_{i}} s_{j}^{i}\left(\kappa r_{\varepsilon}\right)^{-j}+s_{0}^{i} \ln \kappa r_{\varepsilon}\right)$,

where $\delta_{i}=2 i+1$ in the static scenario and $\delta_{i}=3 i+1$ in the free-fall scenario. Although we calculated the atmosphere entropy around the 4D black hole to $\mathcal{O}\left(m_{p}^{-2}\right)$ in the freefall scenario, the $2 \mathrm{D}$ black hole result suggests that Eq. (78) might hold to all orders in this scenario. For $s_{1}^{0}$ and $s_{0}^{0}$ in Eq. (78), we find that

$s_{1}^{0}=\frac{A \kappa^{2}}{720 \pi}$,

$s_{0}^{0}=-\frac{C\left(r_{h}^{2}\right) \kappa^{2}}{180}\left(c_{1}-2 \eta\right)$,

where $A=4 \pi C\left(r_{h}^{2}\right)$ is the horizon area. In the nondispersive scenario $\left(m_{p} \rightarrow \infty\right)$, the terms $\frac{s_{1}^{0}}{\kappa r_{\varepsilon}}$ and $s_{0}^{0} \ln \kappa r_{\varepsilon}$ are the usual leading and subleading logarithmic divergent terms, respectively. Note that $s_{1}^{0}$ and $s_{0}^{0}$ have already been calculated in the non-dispersive scenario [44-46].

It seems from Eq. (78) that the near horizon divergence of the atmosphere entropy gets worse for the higher order corrections in the MDR as $\kappa r_{\varepsilon} \rightarrow 0$. However, the higher order contributions in Eq. (78) are always accompanied with powers of the factor $\frac{T_{0}^{2}}{m_{p}^{2}}=\left(\frac{m_{p} \kappa}{2 \pi}\right)^{2}$. Thus, one might hope that the higher order divergent problem would become less severe if $r_{\varepsilon}$ somehow can be related to $m_{p}$. One way to understand the value of $r_{\varepsilon}$ is by introducing the proper length for $r_{\varepsilon}$ as

$\varepsilon=\int_{r_{h}}^{r_{h}+r_{\varepsilon}} \sqrt{g_{r r}} \mathrm{~d} r$.

The brick wall is put at $r=r_{h}+r_{\varepsilon}$ to cut off the unknown quantum physics of gravity. In this sense, the invariant distance of the wall from the horizon $\varepsilon$ could be given by $\varepsilon \sim m_{p}$. Thus, we could define $\alpha$ such as $\varepsilon=\alpha m_{p}$. Indeed in the 't Hooft's original calculation, equating $\frac{s_{1}^{0}}{\kappa r_{\varepsilon}}$ to the black hole's Bekenstein-Hawking entropy $S_{B H}=\frac{A}{4 m_{p}^{2}}$ gives

$r_{\varepsilon}=\frac{\kappa m_{p}^{2}}{180 \pi}$ 
Note that Eq. (80) depends on the chosen coordinate system. In the scenario without the MDR, a natural choice is that $\varepsilon$ is measured along a static time slice. Thus, Eq. (80) is calculated in the Schwarzschild-like coordinate [44]. Assuming $r_{\varepsilon} \ll r_{h}$, one finds from Eq. (80)

$r_{\varepsilon} \approx \frac{\kappa \varepsilon^{2}}{2}$.

Thus, Eq. (81) gives

$\alpha=\sqrt{\frac{1}{90 \pi}}$,

where we reproduce 't Hooft's result.

In the static scenario, it is still natural to assume that $\varepsilon$ is measured along a static time slice. If we let $\varepsilon=\alpha m_{p}$ in Eq. (78), we find the atmosphere entropy around the horizon becomes

$S \sim \frac{A}{4 m_{p}^{2}} \tilde{s}_{0}+2 s_{0}^{0} \ln \kappa m_{p}+$ Finite terms as $m_{p} \kappa \rightarrow 0$,

where $\tilde{s}_{0}$ was given in [39]. The leading divergent coefficient $\tilde{s}_{0}$ is determined by the coefficients $\tilde{C}_{n}$ in the MDR (67) and $f_{j}^{n}$ and $c_{i}^{a}$, which are defined in Eq. (55). For a general black hole, $f_{j}^{n}$ and $c_{i}^{a}$ could depend on the parameters of the black hole. However, they are pure numbers for a Schwarzschild black hole. Thus, for a Schwarzschild black hole, $\tilde{s}_{0}$ does not depend on the black hole's properties and the leading divergent term in Eq. (84) scales with the horizon area $A$.

In the free-fall scenario, one might prefer that the proper length $\varepsilon$ is measured on a time slice orthogonal to the freefall world lines [49]. In this case, Eq. (80) for $\varepsilon$ should be calculated in the PG coordinate and one then has $\varepsilon=r_{\varepsilon}$. If one has $\varepsilon=\alpha m_{p}$, the entropy of the atmosphere around the horizon becomes

$$
\begin{aligned}
S & \sim \frac{A \kappa}{720 \pi \alpha m_{p}}+s_{0}^{0} \ln \kappa m_{p} \\
& +\sum_{l=1}^{\infty} \frac{s_{l}}{\left(m_{p} \kappa\right)^{l}}+\text { Finite terms as } m_{p} \kappa \rightarrow 0
\end{aligned}
$$

where we define $s_{l}=\sum_{i=\max \{1, l-1\}}^{\infty} \frac{s_{l+2 i}^{i}}{(2 \pi)^{2 i} \alpha^{l+2 i}}$. Moreover, Eq. (85) might suggest that the effects of the MDR on the atmosphere entropy is nonperturbative in this case. Alternatively, inspired by the static scenario, one could choose $r_{\varepsilon}$ such that the higher order terms in Eq. (78) have the same order of divergence as $\frac{1}{\kappa r_{\varepsilon}}$. Here, we could have

$r_{\varepsilon}=\alpha m_{p}^{\frac{2}{3}} \kappa^{-\frac{1}{3}}$,

where $\alpha$ is some constant. In this case, the atmosphere entropy becomes
$S \sim \frac{\tilde{s}_{0}}{\alpha\left(m_{p} \kappa\right)^{\frac{2}{3}}}+\frac{2 s_{0}^{0}}{3} \ln m_{p} \kappa+$ Finite terms as $m_{p} \kappa \rightarrow 0$,

where we define

$\tilde{s}_{0}=s_{1}^{0}\left[1+\sum_{i=1}^{\infty} \frac{s_{3 i+1}^{i}}{s_{1}^{0}}\left(2 \pi \alpha^{\frac{3}{2}}\right)^{-2 i}\right]$.

For a Schwarzschild black hole, the terms in the square bracket in Eq. (88) do not depend on the black hole's properties and the leading divergent term in Eq. (87) scales with $A \kappa^{\frac{4}{3}} m_{p}^{-\frac{2}{3}}$. In [49], the authors calculated the black hole horizon entanglement entropy for a massless scalar field with the MDR imposed in a free-fall frame. With the sub- or superluminal dispersion with index $n$, they found that the entanglement entropy scales as $A \kappa^{1+\frac{1}{n}} m_{p}^{-1+\frac{1}{n}}$.

Following the argument proposed in [50], the authors in [51] obtained modified relations between the mass of a Schwarzschild black hole and its temperature and entropy. The argument connecting a MDR and some modifications of the entropy of black holes is formulated in a scheme of analysis first introduced by Bekenstein [42]. In fact, for the MDR in Eq. (1), the modified temperature of the black hole was given by

$T_{\mathrm{eff}}=\frac{1}{4 \pi} F\left(\frac{m_{p}^{2}}{2 M}\right)$

where $M$ is the mass of the black hole. The first law of black hole thermodynamics $\mathrm{d} S_{B}=\frac{d M}{T}$ and Eq. (2) lead to the modified entropy of the black hole

$S_{B}=\frac{A}{4 m_{p}^{2}}+2 \pi C_{1} \ln \kappa m_{p}+$ Finite terms as $m_{p} \kappa \rightarrow 0$,

where $A=16 \pi M^{2}$ and $\kappa=\frac{1}{4 M}$. It is interesting to note that the modifications of the entropy of black holes in Eq. (90) are finite as $m_{p} \kappa \rightarrow 0$ except the logarithmic term. If one wants the same story for the atmosphere entropy as obtained in Eq. (78), one could have $r_{\varepsilon}=\alpha \kappa^{\delta-1} m_{p}^{\delta}$ for some constant $\alpha$ where $0<\delta \leq \frac{2}{3}$ in the static scenario and $0<\delta \leq \frac{1}{2}$ in the free-fall scenario. Hence, the atmosphere entropy becomes

$S \sim \frac{s_{1}^{0}}{\alpha\left(\kappa m_{p}\right)^{\delta}}+s_{0}^{0} \delta \ln \kappa m_{p}+$ Finite terms as $m_{p} \kappa \rightarrow 0$,

where the leading divergent term scales with $A \kappa^{2-\delta} m_{p}^{-\delta}$ for a Schwarzschild black hole. The coefficient of the logarithmic term in Eq. (90) depends on $C_{1}$, a coefficient of the MDR. However, we show that the coefficient of the subleading logarithmic term in the atmosphere entropy is irrelevant to the 
coefficients of the MDR. It only depends on the position of the wall, $r_{\varepsilon}$ and the properties of the black hole.

For a 2D black hole with the Schwarzschild-like coordinate

$\mathrm{d} s^{2}=f(r) \mathrm{d} t^{2}-\frac{\mathrm{d} r^{2}}{f(r)}$,

the atmosphere entropy of a massless scalar can also be presented in the form of a Laurent series with respect to $r_{\varepsilon}$

$S \sim s_{0}^{0} \ln \kappa r_{\varepsilon}+\sum_{i=1}^{\infty} \frac{T_{0}^{2 i}}{m_{p}^{2 i}}\left(\sum_{j=1}^{\delta_{i}} s_{j}^{i}\left(\kappa r_{\varepsilon}\right)^{-j}+s_{0}^{i} \ln \kappa r_{\varepsilon}\right)$,

where $\delta_{i}=2 i$ in the static scenario and $\delta_{i}=3 i$ in the freefall scenario. From Eqs. (64) and (84), one has $s_{0}^{0}=-\frac{1}{12}$. In the static scenario, if we assume that the proper length $\varepsilon$ is measured along a static time slice and $\varepsilon=\alpha m_{p}$, the atmosphere entropy of a massless scalar becomes

$S \sim-\frac{1}{6} \ln \kappa m_{p}+$ Finite terms as $\kappa m_{p} \rightarrow 0$,

where the same leading logarithmic term was also obtained in [52] for the scenario without the MDR. In the free-fall scenario, if the proper length $\varepsilon$ is assumed to be measured on a time slice orthogonal to the free-fall world lines and we let $\varepsilon=\alpha m_{p}$, the atmosphere entropy of a massless scalar becomes

$S \sim-\frac{1}{12} \ln \kappa m_{p}+\frac{1}{4 \pi^{2}} \sum_{n=1}^{\infty} \frac{s_{n}}{\left(\kappa m_{p}\right)^{n}}$

+ Finite terms as $\kappa m_{p} \rightarrow 0$,

where $s_{n}$ can be determined by Eq. (64). If we want that the modifications of the entropy of black holes in Eq. (93) are finite, we could have $r_{\varepsilon}=\alpha \kappa^{\delta-1} m_{p}^{\delta}$ for some constant $\alpha$ where $0<\delta \leq \frac{2}{3}$ for some constant $\alpha$. The entropy then becomes

$S \sim-\frac{\delta}{12} \ln \kappa m_{p}+$ Finite terms as $\kappa m_{p} \rightarrow 0$.

\section{Black hole evaporation}

In the section, we discuss the MDR effects on the evaporations of a Schwarzschild black hole to $\mathcal{O}\left(\frac{T_{0}^{2}}{m_{p}^{2}}\right)$ in the freefall scenario. For simplicity, we assume that the emitted particles are massless. In [53], Page counted the number of modes per frequency interval with periodic boundary conditions in a large container around the black hole and divided it by the time it takes a particle to cross the container. He then related the expected number emitted per mode $n_{\omega, l}$ to the average emission rate per frequency interval $\frac{\mathrm{d} n_{\omega, l}}{\mathrm{~d} t}$ by $\frac{\mathrm{d} n_{\omega, l}}{\mathrm{~d} t}=n_{\omega, l} \frac{\mathrm{d} \omega}{2 \pi \hbar}$,

for each mode and frequency interval $(\omega, \omega+\mathrm{d} \omega)$. Following the same argument, we find that in the MDR case

$\frac{\mathrm{d} n_{\omega, l}}{\mathrm{~d} t}=n_{\omega, l} \frac{\partial \omega}{\partial p_{r}} \frac{\mathrm{d} p_{r}}{2 \pi \hbar}=n_{\omega, l} \frac{\mathrm{d} \omega}{2 \pi \hbar}$,

where $\frac{\partial \omega}{\partial p_{r}}$ is the radial velocity of the particle and the number of modes between the wavevector interval $\left(p_{r}, p_{r}+\mathrm{d} p_{r}\right)$ is $\frac{\mathrm{d} p_{r}}{2 \pi \hbar}$. Since each particle carries off the energy $\omega$, the total luminosity is obtained from $\frac{\mathrm{d} n_{\omega, l}}{\mathrm{~d} t}$ by multiplying by the energy $\omega$ and summing up over all energy $\omega$ and $l$,

$L=\sum_{l=0}(2 l+1) \int \omega n_{\omega, l} \frac{\mathrm{d} \omega}{2 \pi \hbar}$.

However, some of the radiation emitted by the horizon might not be able to reach the asymptotic region. Before the radiation reaches the distant observer, the curved spacetime around the black hole horizon, which plays the role of a potential barrier, must be passed. This effect on $L$ can be described by a graybody factor from the scattering coefficients of the black hole. Actually, the graybody factor is given by $\left|T_{l}(\omega)\right|^{2}$, where $T_{l}(\omega)$ represents the transmission coefficient of the black hole barrier which in general depends on the energy $\omega$ and angular momentum $l$ of the particle. Taking the graybody factor into account, we find for the total luminosity

$L=\sum_{l=0}(2 l+1) \int\left|T_{l}(\omega)\right|^{2} \omega n_{\omega, l} \frac{\mathrm{d} \omega}{2 \pi \hbar}$.

The relevant radiation usually has the energy of order $\hbar M^{-1}$ for a black hole with the mass $M$, one hence needs to use the wave equations given in the appendix of [39] to compute $\left|T_{l}(\omega)\right|^{2}$ accurately. However, solving the wave equations for $\left|T_{l}(\omega)\right|^{2}$ could be very complicated. On the other hand, we can use the geometric optics approximation to estimate $\left|T_{l}(\omega)\right|^{2}$. In the geometric optics approximation, we assume $\omega \gg M$ and the high energy waves will be absorbed unless they are aimed away from the black hole. Hence $\left|T_{l}(\omega)\right|^{2}=1$ for all the classically allowed energy $\omega$ and the angular momentum $l$, while $\left|T_{i}(E)\right|^{2}=0$ otherwise. For the usual dispersion relation, the well-known Stefan law for black holes is obtained in this approximation.

To find the classically allowed angular momentum $l$ with fixed value of energy $\omega$, we consider Eq. (6) for a massless particle in the Schwarzschild black hole with the mass $M$. Solving Eq. (6) for $\lambda$ to $\mathcal{O}\left(m_{p}^{-2}\right)$ gives

$\frac{\lambda}{r^{2}}=\left(\omega-v p_{r}\right)^{2}-\frac{2 C_{1}}{m_{p}^{2}}\left(\omega-v p_{r}\right)^{4}-p_{r}^{2}$, 
where we have $\lambda=\left(l+\frac{1}{2}\right)^{2} \hbar^{2}, v(r)=-\sqrt{\frac{2 M}{r}}$, and $C_{1}$ is given in Eq. (1). In the geometric optics approximation, $p_{r}$ is always a real number. In the non-dispersive case $\left(m_{p} \rightarrow \infty\right)$, the maximum of the RHS of Eq. (101) is $\frac{\omega^{2}}{1-\frac{2 M}{r}}$. Thus, one has

$\lambda \leq \frac{r^{2} \omega^{2}}{1-\frac{2 M}{r}}$,

where the RHS has a minimum at $r_{\min }=3 M$, which is $27 M^{2} \omega^{2}$. If the particles overcome the angular momentum barrier and get absorbed by the black hole, one must have $\lambda \leq 27 M^{2} \omega^{2}$. In the geometric optics approximation, the Schwarzschild black hole is just like a black sphere of radius $R=3^{3 / 2} M$ [54]. When the second term in the RHS of Eq. (101) is included, the maximum of the RHS is shifted to

$\Omega \equiv \frac{\omega^{2}}{1-\frac{2 M}{r}}-\frac{2 C_{1} \omega^{2}}{m_{p}^{2}} \frac{\omega^{2}}{\left(1-\frac{2 M}{r}\right)^{4}}$.

The minimum of $r^{2} \Omega$ is then shifted to

$\lambda_{\max } \equiv 27 M^{2} \omega^{2}\left(1-\frac{54 C_{2} \omega^{2}}{m_{p}^{2}}\right)$.

Therefore, the particle must have $\lambda \leq \lambda_{\max }$ to get absorbed by the black hole. Equation (100) then becomes

$L=\int \frac{\omega \mathrm{d} \omega}{2 \pi \hbar^{3}} \int_{0}^{\lambda_{\max }} n\left(\frac{\omega(1+\Delta)}{T_{0}}\right) \mathrm{d} \lambda$,

where Eq. (41) for $n_{\omega, l}$ is used. For a massless particle in the Schwarzschild black hole, Eq. (24) gives

$\Delta=-\frac{C_{1}}{2 m_{p}^{2}}\left(20 \omega^{2}-\frac{3 \lambda}{4 M^{2}}\right)$.

Expanding $n\left(\frac{\omega(1+\Delta)}{T_{0}}\right)$ to $\mathcal{O}\left(m_{p}^{-2}\right)$ and then integrating Eq. (105), we find for the emission of $n_{s}$ species of massless scalars and $n_{f}$ species of massless spin- $1 / 2$ fermions that the total luminosity is

$$
\begin{aligned}
L= & \frac{9 m_{p}^{2}}{40960 M^{2}}\left[\left(n_{s}+\frac{7}{4} n_{f}\right)-C_{1}\left(0.73 n_{s}+1.41 n_{f}\right) \frac{m_{p}^{2}}{M^{2}}\right. \\
& \left.+\mathcal{O}\left(\frac{m_{p}^{3}}{M^{3}}\right)\right] .
\end{aligned}
$$

To make a comparison with the static scenario, the results in [39] are given below for the form of MDR (1). In the static scenario, the correction to the Hawking temperature is

$$
\Delta=-\frac{C_{1}}{2 m_{p}^{2}}\left(4 \omega^{2}+\frac{\lambda}{4 M^{2}}\right),
$$

the maximum of the angular momentum is

$\lambda_{\max }=27 M^{2} \omega^{2}\left(1-\frac{6 C_{1} \omega^{2}}{m_{p}^{2}}\right)$,

and the total luminosity is

$$
\begin{aligned}
L= & \frac{9 m_{p}^{2}}{40960 \pi M^{2}}\left[\left(n_{s}+\frac{7}{4} n_{f}\right)+C_{1}\left(0.48 n_{s}+0.92 n_{f}\right) \frac{m_{p}^{2}}{M^{2}}\right. \\
& \left.+\mathcal{O}\left(\frac{m_{p}^{3}}{M^{3}}\right)\right] .
\end{aligned}
$$

Note that the sign in front of $C_{1}$ in Eq. (107) is different from that in Eq. (110). For the sub-luminal dispersion relation with $C_{1}<0$, it means that the total luminosity increases due to the MDR effects in the free-fall scenario, while it decreases in the static scenario. In the geometric optics approximation, the black hole can be described as a black sphere for absorbing particles. The total luminosity is determined by the radius of the black sphere $R$ and the temperature of the black hole $T$. Note that $R=\sqrt{\frac{\lambda_{\max }}{\omega^{2}}}$ and $T_{\text {eff }} \approx T_{0}(1-\Delta)$. In the static scenarios, the MDR effects increase the radius of the black sphere, while they decrease the temperature of the black hole. The competition between the increased radius and the decreased temperature determines whether the luminosity would increase or decrease. It appears from Eq. (110) that the effects of the decreased temperature win the competition. In the free-fall scenario, the MDR effects also increase the radius of the black sphere. Due to the minus sign in front of $\frac{3 \lambda}{4 M^{2}}$ in Eq. (106), the temperature of the black hole increases for $\lambda>\frac{80}{3} M^{2} \omega^{2}$ and decreases for $\lambda<\frac{80}{3} M^{2} \omega^{2}$, but more slowly than in the static scenario. As a result, the effects of the increased radius win the competition and hence the luminosity increases. The opposite story occurs in the super-luminal case with $C_{1}>0$.

\section{Conclusion}

In this paper, we used the Hamilton-Jacobi method to calculate the tunneling rates of radiations across the horizon and the effective Hawking temperatures in the dispersive models with the free-fall preferred frame. After the near horizon spectrum of the radiation was obtained, the thermal entropy of radiations near the horizon and the luminosity of the black hole were computed. Our main results are:

- In Sect. 2, we first derived the deformed Hamilton-Jacobi equations in the dispersive models with the free-fall preferred frame. The deformed Hamilton-Jacobi equations were then solved for $\partial_{r} I$ and the imaginary part of $I$ was obtained. The corrections to the Hawking temperature were calculated for massive and charged particles to 
$\mathcal{O}\left(m_{p}^{-2}\right)$ and neutral and massless particles with $\lambda=0$ to all orders, respectively. It was found that the corrections were suppressed by $m_{p}$.

- In Sect. 3, we used the brick wall model to compute the thermal entropy of a massless scalar field near the horizon of a 4D spherically symmetric black hole and a 2D one. For a 4D black hole, the entropy near the horizon have been calculated to $\mathcal{O}\left(m_{p}^{-2}\right)$ and could be written in the form of Eq. (78). The entropy became divergent as the wall approached the horizon. Various choices of the proper distance between the wall and the horizon and the corresponding entropies have been discussed. For a 2D black hole, entropies in the static and free-fall scenarios have been calculated to all orders. The leading divergent terms were logarithmic. Nevertheless, their coefficients depended on choices of the proper distance between the wall and the horizon.

- In Sect. 4, we calculated the luminosities of a Schwarzschild black hole with the mass $M \gg m_{p}$. We used the geometric optics approximation to estimate the effects of scattering off the background. A comparison between the static scenario and the free-fall one has been given there.

Acknowledgments We are grateful to Houwen Wu and Zheng Sun for useful discussions. This work is supported in part by NSFC (Grant Nos. 11005016, 11175039 and 11375121) and the Fundamental Research Funds for the Central Universities.

Open Access This article is distributed under the terms of the Creative Commons Attribution 4.0 International License (http://creativecomm ons.org/licenses/by/4.0/), which permits unrestricted use, distribution, and reproduction in any medium, provided you give appropriate credit to the original author(s) and the source, provide a link to the Creative Commons license, and indicate if changes were made.

Funded by SCOAP ${ }^{3}$.

\section{References}

1. S.W. Hawking, Particle creation by black holes. Commun. Math. Phys. 43, 199 (1975) [Erratum-ibid. 46, 206 (1976)]

2. W.G. Unruh, Notes on black hole evaporation. Phys. Rev. D 14, 870 (1976)

3. W.G. Unruh, Sonic analog of black holes and the effects of high frequencies on black hole evaporation. Phys. Rev. D 51, 2827 (1995)

4. R. Brout, S. Massar, R. Parentani, P. Spindel, Hawking radiation without transPlanckian frequencies. Phys. Rev. D 52, 4559 (1995). arXiv:hep-th/9506121

5. S. Corley, T. Jacobson, Hawking spectrum and high frequency dispersion. Phys. Rev. D 54, 1568 (1996). arXiv:hep-th/9601073

6. S. Corley, Computing the spectrum of black hole radiation in the presence of high frequency dispersion: an analytical approach. Phys. Rev. D 57, 6280 (1998). arXiv:hep-th/9710075

7. Y. Himemoto, T. Tanaka, A generalization of the model of Hawking radiation with modified high frequency dispersion relation. Phys. Rev. D 61, 064004 (2000). arXiv:gr-qc/9904076

8. H. Saida, M.A. Sakagami, Black hole radiation with high frequency dispersion. Phys. Rev. D 61, 084023 (2000). arXiv:gr-qc/9905034
9. W.G. Unruh, R. Schutzhold, On the universality of the Hawking effect. Phys. Rev. D 71, 024028 (2005). arXiv:gr-qc/0408009

10. J. Macher, R. Parentani, Black/White hole radiation from dispersive theories. Phys. Rev. D 79, 124008 (2009). arXiv:0903.2224 [hepth]

11. A. Coutant, R. Parentani, S. Finazzi, Black hole radiation with short distance dispersion, an analytical S-matrix approach. Phys. Rev. D 85, 024021 (2012). arXiv:1108.1821 [hep-th]

12. A. Coutant, R. Parentani, Hawking radiation with dispersion: the broadened horizon paradigm. Phys. Rev. D 90(12), 121501 (2014). arXiv: 1402.2514 [gr-qc]

13. F. Belgiorno, S.L. Cacciatori, F.D. Piazza, Tunneling approach and thermality in dispersive models of analogue gravity. arXiv:1411.7871 [gr-qc]

14. C. Barcelo, S. Liberati, M. Visser, Analogue gravity. Living Rev. Relativ. 8, 12 (2005) [Living Rev. Rel. 14, 3 (2011)] arXiv:gr-qc/0505065

15. T. Jacobson, Trans Planckian redshifts and the substance of the space-time river. Prog. Theor. Phys. Suppl. 136, 1 (1999). arXiv:hep-th/0001085

16. P. Kraus, F. Wilczek, Selfinteraction correction to black hole radiance. Nucl. Phys. B 433, 403 (1995). arXiv:gr-qc/9408003

17. P. Kraus, F. Wilczek, Effect of selfinteraction on charged black hole radiance. Nucl. Phys. B 437, 231 (1995). arXiv:hep-th/9411219

18. K. Srinivasan, T. Padmanabhan, Particle production and complex path analysis. Phys. Rev. D 60, 024007 (1999). arXiv:gr-qc/9812028

19. M. Angheben, M. Nadalini, L. Vanzo, S. Zerbini, Hawking radiation as tunneling for extremal and rotating black holes. JHEP 0505 , 014 (2005). arXiv:hep-th/0503081

20. R. Kerner, R.B. Mann, Tunnelling, temperature and Taub-NUT black holes. Phys. Rev. D 73, 104010 (2006). arXiv:gr-qc/0603019

21. S. Hemming, E. Keski-Vakkuri, The spectrum of strings on BTZ black holes and spectral flow in the SL(2, R) WZW model. Nucl. Phys. B 626, 363 (2002). arXiv:hep-th/0110252

22. A.J.M. Medved, Radiation via tunneling from a de Sitter cosmological horizon. Phys. Rev. D 66, 124009 (2002). arXiv:hep-th/0207247

23. E.C. Vagenas, Semiclassical corrections to the BekensteinHawking entropy of the BTZ black hole via selfgravitation. Phys. Lett. B 533, 302 (2002). arXiv:hep-th/0109108

24. M. Arzano, A.J.M. Medved, E.C. Vagenas, Hawking radiation as tunneling through the quantum horizon. JHEP 0509, 037 (2005). arXiv:hep-th/0505266

25. S.Q. Wu, Q.Q. Jiang, Remarks on Hawking radiation as tunneling from the BTZ black holes. JHEP 0603, 079 (2006). arXiv:hep-th/0602033

26. M. Nadalini, L. Vanzo, S. Zerbini, Hawking radiation as tunneling: the D dimensional rotating case. J. Phys. A 39, 6601 (2006). arXiv:hep-th/0511250

27. B. Chatterjee, A. Ghosh, P. Mitra, Tunnelling from black holes in the Hamilton Jacobi approach. Phys. Lett. B 661, 307 (2008). arXiv:0704.1746 [hep-th]

28. V. Akhmedova, T. Pilling, A. de Gill, D. Singleton, Temporal contribution to gravitational WKB-like calculations. Phys. Lett. B 666, 269 (2008). arXiv:0804.2289 [hep-th]

29. E.T. Akhmedov, T. Pilling, D. Singleton, Subtleties in the quasiclassical calculation of Hawking radiation. Int. J. Mod. Phys. D 17, 2453 (2008). arXiv:0805.2653 [gr-qc]

30. V. Akhmedova, T. Pilling, A. de Gill, D. Singleton, Comments on anomaly versus WKB/tunneling methods for calculating Unruh radiation. Phys. Lett. B 673, 227 (2009). arXiv:0808.3413 [hep-th]

31. R. Banerjee, B.R. Majhi, Quantum tunneling and back reaction. Phys. Lett. B 662, 62 (2008). arXiv:0801.0200 [hep-th]

32. D. Singleton, E.C. Vagenas, T. Zhu, J.R. Ren, Insights and possible resolution to the information loss paradox via the tunneling 
picture. JHEP 1008, 089 (2010) [Erratum-ibid. 1101, 021 (2011)] arXiv: 1005.3778 [gr-qc]

33. D. Chen, H. Wu, H. Yang, Fermion's tunnelling with effects of quantum gravity. Adv. High Energy Phys. 2013, 432412 (2013). arXiv:1305.7104 [gr-qc]

34. D. Chen, H. Wu, H. Yang, Observing remnants by fermions' tunneling. JCAP 1403, 036 (2014). arXiv:1307.0172 [gr-qc]

35. D.Y. Chen, Q.Q. Jiang, P. Wang, H. Yang, Remnants, fermions“ tunnelling and effects of quantum gravity. JHEP 1311, 176 (2013). arXiv:1312.3781 [hep-th]

36. D. Chen, Z. Li, Remarks on remnants by fermions' tunnelling from black strings. Adv. High Energy Phys. 2014, 620157 (2014). arXiv: 1404.6375 [hep-th]

37. D. Chen, H. Wu, H. Yang, S. Yang, Int. J. Mod. Phys. A 29(26), 1430054 (2014). arXiv: 1410.5071 [gr-qc]

38. B. Mu, P. Wang, H. Yang, Minimal length effects on tunnelling from spherically symmetric black holes. Adv. High Energy Phys. 2015, 898916 (2015). arXiv: 1501.06025 [gr-qc]

39. P. Wang, H. Yang, Black Hole Radiation with Modified Dispersion Relation in Tunneling Paradigm: Static Frame. arXiv:1505.03045 [gr-qc]

40. S. Corley, T. Jacobson, Lattice black holes. Phys. Rev. D 57, 6269 (1998). arXiv:hep-th/9709166

41. J.D. Bekenstein, Black holes and the second law. Lett. Nuovo Cim. 4, 737 (1972)

42. J.D. Bekenstein, Black holes and entropy. Phys. Rev. D 7, 2333 (1973)

43. S.W. Hawking, Black holes and thermodynamics. Phys. Rev. D 13, $191(1976)$

44. G. 't Hooft, On the quantum structure of a black hole. Nucl. Phys. B 256, 727 (1985)
45. S.N. Solodukhin, The conical singularity and quantum corrections to entropy of black hole. Phys. Rev. D 51, 609 (1995). arXiv:hep-th/9407001

46. S.N. Solodukhin, Entanglement entropy of black holes. Living Rev. Relativ. 14, 8 (2011). arXiv:1104.3712 [hep-th]

47. B.D. Chowdhury, Problems with tunneling of thin shells from black holes. Pramana 70, 593 (2008) [Pramana 70, 3 (2008)] arXiv:hep-th/0605197

48. J.B. Hartle, S.W. Hawking, Path integral derivation of black hole radiance. Phys. Rev. D 13, 2188 (1976)

49. T. Jacobson, R. Parentani, Black hole entanglement entropy regularized in a freely falling frame. Phys. Rev. D 76, 024006 (2007). arXiv:hep-th/0703233

50. G. Amelino-Camelia, M. Arzano, A. Procaccini, Severe constraints on loop-quantum-gravity energy-momentum dispersion relation from black-hole area-entropy law. Phys. Rev. D 70, 107501 (2004). arXiv:gr-qc/0405084

51. G. Amelino-Camelia, M. Arzano, Y. Ling, G. Mandanici, Blackhole thermodynamics with modified dispersion relations and generalized uncertainty principles. Class. Quantum Gravity 23, 2585 (2006). arXiv:gr-qc/0506110

52. R.B. Mann, L. Tarasov, A. Zelnikov, Brick walls for black holes. Class. Quantum Gravity 9, 1487 (1992)

53. D.N. Page, Particle emission rates from a black hole: massless particles from an uncharged, nonrotating hole. Phys. Rev. D 13, 198 (1976)

54. R.M. Wald, General Relativity (University of Chicago Press, Chicago, 1984), p. 491 